Relations industrielles

Industrial Relations

\title{
Les normes internationales du travail, Genève, Bureau international du travail, Manuel d'éducation ouvrière, 1978, 103 pp.
}

\section{Kim Chi Tran Van}

\section{Volume 34, numéro 1, 1979}

URI : https://id.erudit.org/iderudit/028951ar

DOI : https://doi.org/10.7202/028951ar

Aller au sommaire du numéro

Éditeur(s)

Département des relations industrielles de l'Université Laval

ISSN

0034-379X (imprimé)

1703-8138 (numérique)

Découvrir la revue

Citer ce compte rendu

Tran Van, K. C. (1979). Compte rendu de [Les normes internationales du travail, Genève, Bureau international du travail, Manuel d'éducation ouvrière, 1978, 103 pp.] Relations industrielles / Industrial Relations, 34(1), 206-207.

https://doi.org/10.7202/028951ar

Tous droits réservés (C Département des relations industrielles de l'Université Laval, 1979
Ce document est protégé par la loi sur le droit d'auteur. L’utilisation des services d'Érudit (y compris la reproduction) est assujettie à sa politique d'utilisation que vous pouvez consulter en ligne.

https://apropos.erudit.org/fr/usagers/politique-dutilisation/ 
prétations divergentes - et, d'un côté, de grandes angoisses - sur le maintien du poids traditionnel de la négociation collective au centre du système britannique de relations industrielles. Ce qui ne saurait, encore une fois, étonner en pays nordaméricain...

Ceci, explique excellemment S. Sciarri, parce qu'il est difficile de se détacher d'une vision claire d'autonomie et d'opposition des parties impliquées dans une négociation collective pour emprunter une voie le long de laquelle la coopération entre les dites parties pourrait graduellement éroder la fonction de contestation des décisions entrepreneuriales, apanage traditionnel des syndicats de travailleurs.

Ce qui est vrai pour la Grande Bretagne (Wedderburn), dont le mouvement syndical a construit, grâce à sa propre force, une structure volontaire de négociation collective, non régulée par la loi, mais également pour l'Italie, dont la tradition conflictuelle du syndicat a toujours inséré la négociation collective dans une fonction contestatrice, en excluant toute référence à des solutions autres que conflictuelles.

Le livre paru par les soins de Silvana Sciarra, et dont le sous-titre est « Cogestion, participation, contrôle en Italie et en Europe», rassemble les interventions, en partie revues par leurs auteurs, faites lors d'une journée d'études organisée par l'Institut de Droit du Travail de l'Université de Bari, en juin 1977, sur le thème "La démocratie industrielle en Italie et en Grande Bretagne». K.W. Wedderburn (Lord Wedderburn of Charlton), Cassel Professor of Economics à la London School of Economics and Political Science, était entouré de Gino Giugni, Edoardo Ghera, Federico Mancini et Umberto Romagnoli, tous éminents juristes du travail, du leader syndicaliste C.G.I.L. Bruno Trentin et de l'équipe de Bari, Maria Luisa De Cristofaro, Mario Giovanni Garofalo, Ennio Triggiani, Luciano Garofalo, Gaetano Veneto et Bruno Veneziani (auteur d'une très intéressante communication sur la démocratie industrielle en Suède et en Grande Bretagne), pour ne pas omettre les autres spécialistes, Piepoli, Tucci, Mimola, Biagi.
Colloque stimulant et, à sa façon, complet, dans le signalement duquel on ne peut que passer à regret sur des analyses aussi intéressantes, par exemple, que celles de Wedderburn sur le pouvoir effectif du conseil de (co)surveillance dans les structures sociétaires à double palier ou celles de Giugni sur la frontière mobile de démarcation, dans un pays comme l'Italie, entre l'activité des partis et celle des syndicats, deux réalités distinctes du mouvement ouvrier qui changent, définissent et délimitent leurs tâches réciproques par rapport aux circonstances historiques.

On retiendra, cependant, de ces lignes, je l'espère, qu'on fait un travail de grande qualité à l'Institut de Droit du Travail de l'Université de Bari, et que les relations industrielles italiennes y sont autant cultivées que les relations industrielles internationales et comparées, ce qui dit long sur la vision large et séduisante qu'impriment aux problèmes qui nous préoccupent $\mathrm{Ve}$ neziani, Veneto et $\mathrm{C}^{\circ}$.

Dimitri WEISS

Université de Paris I

Les normes internationales du travail, Genève, Bureau international du travail, Manuel d'éducation ouvrière, 1978, $103 \mathrm{pp}$.

Ce volume portant sur la fonction normative de l'O.I.T. fait partie d'une série de manuels d'éducation ouvrière publiés par l'O.I.T.. Il vise notamment à servir de guide aux moniteurs d'éducation ouvrière, aux responsables syndicaux, aux personnes qui s'intéressent aux droits de l'homme, aux questions du travail et aux questions sociales.

Le premier chapitre retrace les origines, l'historique et la structure de l'organisme international.

Le deuxième chapitre s'intitulant «origine des normes" nous donne un aperçu des premières normes adoptées qui sont en général inspirées par des dirigeants syndicaux.

Le processus d'établissement des normes qui conduit à l'adoption d'une con- 
vention ou d'une recommandation est traité dans le chapitre 3. Un exemple précis a été exposé; ce qui nous permet de comprendre plus facilement le déroulement des étapes nécessaires de la procédure d'adoption d'une norme laquelle devrait être souple pour pouvoir s'adapter à tout pays membre dont les structures sociales sont différentes.

Le chapitre 4, un des chapitre les plus importants, traite des principales raisons, soit la paix universelle et durable fondée sur la base de la justice sociale, la nécessité d'améliorer les conditions de travail existantes et la crainte des répercussions sociales de la concurrence internationale, pour lesquelles devrait être établie une organisation ayant pour but principal l'adoption de norme de travail. Outre les raisons, le chapitre nous fournit le contenu du code international du travail avec toutes les conventions et les recommandations adoptées à ce jour.

Les chapitres 5 , 6 et 7 nous décrivent les procédures de soumission aux États membres d'une convention ou d'une recommandation nouvellement adoptées et les procédures de ratification ou de dénonciation d'une convention par un État membre. Les États membres doivent aussi présenter des rapports sur les conventions non-ratifiées.

Lorsqu'une convention est ratifiée, les gouvernements devraient respectés les obligations dictées par les procédures normales de contrôle de l'O.I.T., qui reposent en général, sur l'hypothèse que les gouvernements agissent de bonne foi et qui font un large appel à la persuasion et à la publicité.

Il existe aussi des procédures spéciales de contrôle qui sont, entre autres, des procédures de réclamation et de plainte en cas de non-observance d'une convention ratifiée.

La liberté syndicale qui est la condition préalable et nécessaire, selon l'O.I.T., à tout progrès vers la justice sociale, est traitée dans le chapitre 8 . Elle occupe une place unique parmi les droits fondamentaux de l'homme dont se préoccupe l'O.I.T.. Par conséquent, l'organisme international institue des procédures spécia- les pour l'examen des plaintes en violation des droits syndicaux.

Enfin, le chapitre 9 expose l'aspect positif de l'influence des normes internationales sur les législations nationales. Souvent, les États membres utilisent les textes des normes internationales comme documentation de base lors de l'élaboration des lois nationales.

À la fin de chaque chapitre, des sujets de discussion sont suggérés. Nous trouvons, en annexe, une liste de conventions et recommandations adoptées et une bibliographie.

Le principal mérite de ce volume est d'avoir su présenter les activités normatives de l'O.I.T. avec des exemples concrets pour illustrer les procédures décrites qui sont, en général, assez complexes. "Les normes internationales du travail» est bien fait et constitue une documentation de base utile pour les personnes qui s'intéressent au domaine des normes de travail.

Kim Chi TRAN VAN

Ministère du Travail et

de la Main-d'Oeuvre

Mediation, an Annotated Bibliography, by E. Levin and D.V. De Santis, Cornell Industrial and Labor Relations Bibliography, Series no. 15, New York State School of Industrial and Labor Relations, Cornell University, Ithaca, New York, 1978, 28 pp.

Cette publication est susceptible d'intéresser à un double point de vue le lecteur canadien qui se préoccupe de l'expérience américaine.

D'une part, il s'agit à notre connaissance d'une première bibliographie écrite sur le sujet au cours des dix dernières années, à tout le moins. Celle-ci a une caractéristique particulière et qui est d'être exhaustive tout en étant sélective. Ainsi, les auteurs ont recensé les divers articles et autres écrits, $y$ incluant des textes de journalistes, qui traitent de la médiation comme sujet principal d'étude. Ils ont négligé les documents qui n'abordent 